\title{
Increased Cholesterol Absorption by Hyperlipidemia Atherosclerosis Prone (LAP) Japanese Quail
}

\author{
Hironori IwASAKI, ${ }^{1}$ Hirosuke OKU, ${ }^{1, *}$ Takayoshi Toda, ${ }^{2}$ Tetsuo NASU, ${ }^{3}$ \\ Tsuyoshi MiYagi $^{1}$ and Isao CHINEN ${ }^{1}$ \\ ${ }^{1}$ Laboratory of Applied Biochemistry, Faculty of Agriculture, University of The Ryukyus, \\ Nishihara-Cho, Okinawa 903-0213, Japan \\ ${ }^{2}$ Department of Clinical Laboratory Medicine, School of Medicine, University of The Ryukyus, \\ Nishihara-Cho, Okinawa 903-0125, Japan \\ ${ }^{3}$ Department of Veterinary Anatomy, Faculty of Agriculture, Miyazaki University, \\ Miyazaki 889-2155, Japan
}

(Received March 27, 2000)

\begin{abstract}
Summary The present study describes the cholesterol absorption by hyperlipidemia atherosclerosis prone (LAP) Japanese quail to address their high susceptibility to experimental atherosclerosis. The apparent cholesterol absorption rate of LAP quail was compared with that of commercially available (CA) Japanese quail. After $14 \mathrm{~d}$ of cholesterol feeding by gavage, it was found that the cholesterol excretion of LAP quail was significantly lower than that of $\mathrm{CA}$ quail. The fecal excretion of bile acid and fat showed a similar tendency to that as shown with the case of cholesterol. The cholesterol feeding only increased the serum cholesterol level of LAP quail, and this trend holds true for the liver lipid concentration. The expression level of liver cholesterol $7 \alpha$-hydroxylase mRNA showed no difference between LAP and CA strains under the conditions of cholesterol loading. These results showed that the cholesterol absorption by LAP quail is significantly higher than that by CA quail, which may reasonably explain the higher susceptibility of this strain to experimental atherosclerosis.
\end{abstract}

Key Words cholesterol feeding, atherosclerosis, absorption, excretion

A strain of Japanese quail susceptible to experimental atherosclerosis (hyperlipidemia atherosclerosis prone: LAP) has been developed by repeated breeding of a highly susceptible line. Our previous study focused on some of the key enzymes of the cholesterol metabolism found comparable activities in LAP and commercially available (CA) quail (1). The lipoprotein and apoprotein profiles were also similar between these two strains (2-4). Thus, the reasons for the higher susceptibility of LAP strain to experimental atherosclerosis are yet to be clarified.

The LAP strain easily develops hypercholesterolemia in response to the dietary loading of cholesterol, and this fact suggests that the input-output balance under atherogenic conditions may be the factor for the increased serum cholesterol level of the LAP strain. The absorption of cholesterol by the small intestine is the first committed step to the cholesterol metabolism. The aim of this study was therefore to compare the apparent cholesterol absorption rate between LAP and CA quail. The expression of cholesterol $7 \alpha$-hydroxylase, the rate limiting enzyme of bile acid production, was studied in relation to the vehicle role of the bile acid for cholesterol absorption.

To minimize the contamination of fecal excretion by

* To whom correspondence should be addressed.

E-mail: b986101@agr.u-ryukyu.ac.jp dietary overspill, cholesterol was administered by use of gavage in this experiment. The fecal excretion was collected throughout the experiment, and the entire inputoutput balance of cholesterol was studied. This experimental feature allowed us, for the first time, to detect the difference in the apparent cholesterol absorption rate between LAP and CA strains.

\section{MATERIALS AND METHODS}

Animals and diets. Male normal Japanese quail (2-3 mo old) were purchased from a commercial supplier (Kyudo Co. Ltd., Kumamoto), and LAP quail (2-3 mo old) were supplied by Tetsuo Nasu, Department of Veterinary Anatomy, Miyazaki University. The animals were randomly assigned to two diet groups $(n=7$ per group), and housed individually in stainless steel cages at a constant room temperature $\left(25^{\circ} \mathrm{C}\right)$ and with a $12 \mathrm{~h}$ light cycle. The quail were fed a commercial diet ad libitum. The indicated composition was $22 \%$ protein, $2.5 \%$ fat, $5 \%$ fiber, $14 \%$ ash, and $2,700 \mathrm{kcal} / \mathrm{kg}$. The animals of the cholesterol feeding groups were fed $50 \mathrm{mg}$ of cholesterol in $500 \mathrm{mg}$ of corn oil by gavage at 14:00 h everyday. The control groups were only fed $500 \mathrm{mg}$ of corn oil. The administration of $50 \mathrm{mg}$ cholesterol and $500 \mathrm{mg}$ corn oil is approximately equal to the daily ingestion of a $10 \mathrm{~g}$ diet containing $0.5 \%$ cholesterol and $5 \%$ corn oil.

Lipid analysis. The blood samples were drawn from 

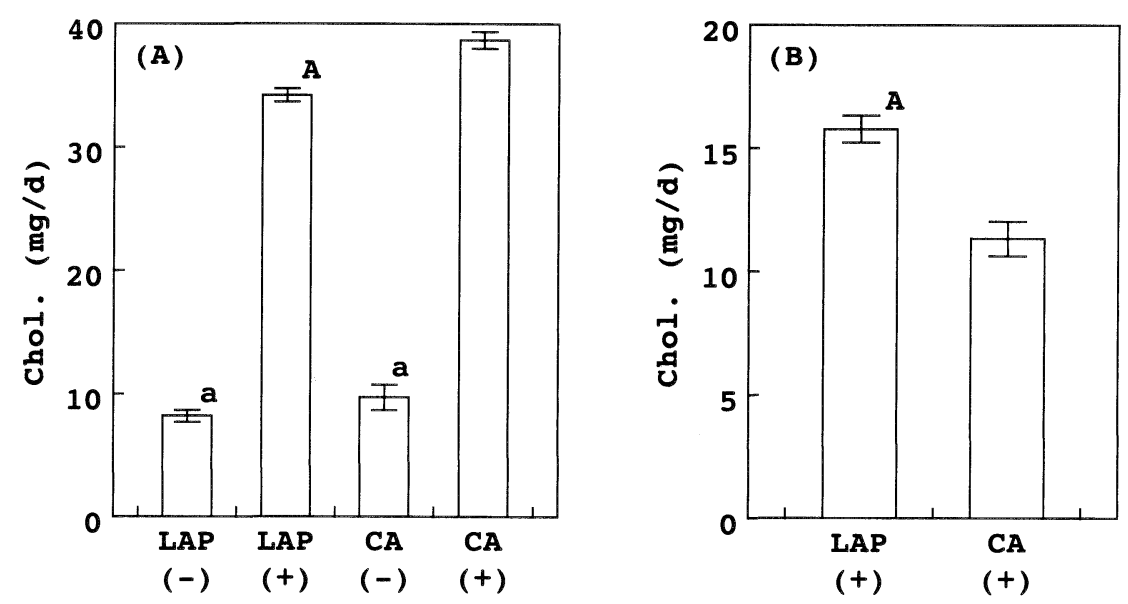

Fig. 1. Fecal cholesterol concentration of LAP and CA quail fed control or cholesterol diets. The cholesterol concentrations in the total feces collected throughout the experiment were analyzed and expressed as excretion per day. A, cholesterol concentration of feces; B, apparent cholesterol absorption of cholesterol-fed quail (cholesterol intake-fecal cholesterol). The apparent absorption rate was $31.6 \%$ for LAP quail and $22.7 \%$ for CA quail. The upper- and lowercase letters above the right corner of the graph bar ( $\mathrm{A}$ and a) respectively show the comparison between strains and diets (cholesterol feeding), and the letter denotes a statistically significant difference at $p<0.05$.

quail fasted overnigth at the end of the experiment, and the liver was excised and stored in a 10\% formaldehyde solution. The feces were collected throughout the experiment onto tin aluminum foil, and entire specimens were lyophilized and kept for analysis at $-20^{\circ} \mathrm{C}$.

The fecal lipids extracted with ethanol at $70^{\circ} \mathrm{C}$ were analyzed enzymatically for cholesterol (5) and bile acids (6). The fecal fatty acids were measured by the method of Van de Kamer et al. (7), and were calculated back as tristearin.

Serum was obtained the centrifugation of the blood at $1,600 \times g$ for $10 \mathrm{~min}$. Serum lipids (cholesterol, triacylglycerol and phospholipids) were analyzed by assay kits supplied by Wako Pure Chemical Industry, Ltd. The liver lipids were extracted with chloroform-methanol $(2 / 1, v / v)$. The concentrations of liver total cholesterol, free cholesterol, triacylglycerol, and phospholipids were determined chemically as described previously $(8-10)$.

Northern blot analysis of hepatic cholesterol $7 \alpha$-hydroxylase mRNA. Total RNA was purified as described previously (2) and used for the quantitative analysis of cholesterol $7 \alpha$-hydroxylase mRNA. The liver total RNA $(0.2 \mu \mathrm{g})$ was denatured in the presence of $6.5 \%$ formaldehyde and $50 \%$ formamide at $55^{\circ} \mathrm{C}$ for $15 \mathrm{~min}$, and electrophoresed in a $1 \%$ agarose gel containing $6.7 \%$ formaldehyde. RNAs were transblotted to a nitrocellulose membrane filter, and cross-linked by baking at $80^{\circ} \mathrm{C}$ for $1 \mathrm{~h}(2)$.

The plasmid $\mathrm{CpcR}^{\mathrm{TM} I I}$ harboring the $\mathrm{CDNA}$ clone of chicken cholesterol $7 \alpha$-hydroxylase was a generous gift of Dr. Furuichi, Professor of Mie University. The ${ }^{32} \mathrm{P}-$ radio-labelled DNA probe directed against the active site of cholesterol $7 \alpha$-hydroxylase was prepared by PCR reaction in the presence of ${ }^{32} \mathrm{P}$-dCTP and the quail liver cDNA as a template. The DNA sequence of the amplicon prepared by this method was $90 \%$ homologous to that of the corresponding region of chicken cholesterol $7 \alpha$ hydroxylase.

The filter was prehybridized with a ${ }^{32} \mathrm{P}$-labelled probe at $68^{\circ} \mathrm{C}$ for $30 \mathrm{~min}$ in $5 \times \mathrm{SSC}, 0.1 \%(\mathrm{w} / \mathrm{v})$ SDS, $5 \%$ $(\mathrm{w} / \mathrm{v})$ dextran sulfate, and 5\%(v/v) blocking reagent (Gene Image ${ }^{\mathrm{TM}}$ random prime labelling module supplied by Amersham International plc). Hybridization was carried out for $16 \mathrm{~h}$ in the same solution containing probe DNA prepared as described above. The membranes were washed successively with $1 \times$ SSC containing $0.1 \%(\mathrm{w} / \mathrm{v}) \mathrm{SDS}$ for $15 \mathrm{~min}$ at $68^{\circ} \mathrm{C}$, and $0.1 \times \mathrm{SSC}$ containing $0.1 \%(\mathrm{w} / \mathrm{v}) \mathrm{SDS}$ for $15 \mathrm{~min}$ at $68^{\circ} \mathrm{C}(11)$.

The blot was exposed to an imaging plate (Fuji Photo Film Co., Ltd.) which integrated and converted the intensity of the radioactivity into graphic images. The spot intensity was measured by computerized analysis of the graphic images. The software used for the measurement was NIH Image (National Institutes of Health).

Statistical analysis. Data were subjected to two-way analysis of variance followed by inspection of the differences between pairs of means by the least significant difference method (12).

\section{RESULTS}

\section{Fecal excretion of cholesterol, fats, and bile acid}

Figure 1A shows the fecal excretion of cholesterol. When the animals were fed the control diet, no significant difference between the LAP $(8.2 \mathrm{mg} / \mathrm{d})$ and CA strains $(9.7 \mathrm{mg} / \mathrm{d})$ was noted in the total fecal cholesterol excretion. With the animals fed the cholesterol diet, the cholesterol excretion for the LAP strain $(34.2 \mathrm{mg} / \mathrm{d})$ was significantly lower than that for the CA strain $(38.7 \mathrm{mg} / \mathrm{d})$. The apparent cholesterol absorption for the LAP and CA strains was therefore 15.8 and $11.3 \mathrm{mg} / \mathrm{d}$, respectively (Fig. 1B). The apparent absorption rate was $31.6 \%$ for LAP and $22.7 \%$ for CA quail. The LAP strain absorbs $4.5 \mathrm{mg}$ of cholesterol more per day than CA quail, and the apparent absorption of the LAP strain was 1.4-fold higher than that of the CA strain. This is the first indication of an overt difference in the absorption rate of cholesterol between LAP and CA strains. 
Table 1. Fecal bile acid and total fat concentrations of LAP and CA quail fed control or cholesterol diets.

\begin{tabular}{|c|c|c|c|c|}
\hline \multirow{2}{*}{ Feces } & \multicolumn{2}{|c|}{ LAP } & \multicolumn{2}{|c|}{$\mathrm{CA}$} \\
\hline & Chol. (-) & Chol. (+) & Chol. (-) & Chol. (+) \\
\hline Bile acid (mg/d) & $2.7 \pm 0.1^{\mathrm{A}}$ & $4.8 \pm 0.7$ & $5.7 \pm 1.4$ & $7.2 \pm 0.5$ \\
\hline Total fat (mg/d) & $68.5 \pm 5.3^{\mathrm{A}}$ & $64.0 \pm 10.6^{\mathrm{A}}$ & $173.1 \pm 20.9$ & $181.5 \pm 12.9$ \\
\hline
\end{tabular}

Data are mean \pm SE of 7 quail. The concentrations of bile acid and total fat in the total feces collected throughout the experiment were analyzed and expressed as excretion per day. The uppercase letter in the table (A) shows the comparison between strains and denotes a statistically significant difference at $p<0.05$.

Table 2. Serum lipid concentrations of LAP and CA quail fed control or cholesterol diets.

\begin{tabular}{|c|c|c|c|c|}
\hline \multirow{2}{*}{ Serum $(m g / d L)$} & \multicolumn{2}{|c|}{ LAP } & \multicolumn{2}{|c|}{$\mathrm{CA}$} \\
\hline & Chol. (-) & Chol. $(+)$ & Chol. (-) & Chol. (+) \\
\hline Total chol. & $219 \pm 23^{\mathrm{a}}$ & $1353 \pm 230^{A}$ & $198 \pm 14$ & $257 \pm 21$ \\
\hline Free chol. & $72.7 \pm 4.4^{\mathrm{a}}$ & $347 \pm 44.7^{\mathrm{A}}$ & $62.6 \pm 3.6$ & $60.9 \pm 4.8$ \\
\hline TG & $109 \pm 8.0^{\mathrm{A}}$ & $137 \pm 14.6^{A}$ & $75.6 \pm 7.1$ & $73.3 \pm 7.5$ \\
\hline PL & $418 \pm 32$ & $558 \pm 58^{\mathrm{A}}$ & $448 \pm 34$ & $432 \pm 45$ \\
\hline
\end{tabular}

Data are mean \pm SE of 7 quail. The upper- and lowercase letters in the table (A and a) respectively show the comparison between strains and diets (cholesterol feeding), and the letter denotes a statistically significant difference at $p<0.05$.

Table 3. Liver lipid concentrations of LAP and CA quail fed control or cholesterol diets.

\begin{tabular}{|c|c|c|c|c|}
\hline \multirow{2}{*}{ Liver (mg/g liver) } & \multicolumn{2}{|c|}{ LAP } & \multicolumn{2}{|c|}{$\mathrm{CA}$} \\
\hline & Chol. (-) & Chol. $(+)$ & Chol. (-) & Chol. $(+)$ \\
\hline Total chol. & $2.99 \pm 0.11^{\mathrm{a}}$ & $14.7 \pm 2.29^{A}$ & $2.81 \pm 0.08$ & $3.04 \pm 0.20$ \\
\hline Free chol. & $2.38 \pm 0.08^{\mathrm{a}}$ & $4.17 \pm 0.13^{A}$ & $2.20 \pm 0.05^{\mathrm{a}}$ & $2.48 \pm 0.10$ \\
\hline $\mathrm{TG}$ & $3.57 \pm 0.41$ & $3.76 \pm 0.54$ & $3.39 \pm 0.38$ & $3.62 \pm 0.66$ \\
\hline PL & $0.39 \pm 0.03$ & $0.42 \pm 0.02$ & $0.39 \pm 0.03$ & $0.43 \pm 0.06$ \\
\hline
\end{tabular}

Data are mean \pm SE of 7 quail. The upper- and lowercase letters in the table (A and a) respectively show the comparison between strains and diets (cholesterol feeding), and the letter denotes a statistically significant difference at $p<0.05$.

The fecal excretion of bile acid and total fat depicted similar trends as shown for cholesterol excretion, suggesting the higher capability of the LAP strain in lipid absorption (Table 1).

Serum cholesterol and lipid concentrations

Table 2 shows the lipid concentrations of serum. In the control group, the serum total and free-cholesterol level were roughly comparable between strains. As has been demonstrated, the serum total and free-cholesterol levels of cholesterol-fed LAP quail were about 5 times higher than those of the CA strain. With respect to CA quail, cholesterol feeding slightly increased the serum cholesterol concentration, but only to a statistically insignificant extent. The concentrations of serum TG and PL were increased in the LAP strain by the cholesterol feeding, but to a lesser extent compared with the case of the cholesterol level.

The profile of liver lipid concentrations manifested essentially the same tendency as shown with the serum lipid levels (Table 3).
Expression of hepatic cholesterol $7 \alpha$-hydroxylase mRNA

The control level of hepatic cholesterol $7 \alpha$-hydroxylase mRNA for LAP quail was 1.5-fold higher than that for CA quail $(p<0.05)$ (Fig. 2). The feeding of cholesterol appeared to decrease the expression of liver cholesterol $7 \alpha$-hydroxylase in LAP quail and increase that in CA quail.

\section{DISCUSSION}

We herein first demonstrated the increased cholesterol absorption of LAP quail compared to the case of CA quail. Although a similar trend in cholesterol absorption was seen with the LAP strain, no statistically significant difference was noted in the previous study, probably due to the contamination of fecal excretion by cholesterol of the dietary overspill (1). The administration of cholesterol by gavage in this study overcame the difficulty encountered in the foregoing experiment and allowed us to demonstrate the increased absorption of cholesterol by the LAP strain for the first time.

On the basis of the fecal excretion of cholesterol and 


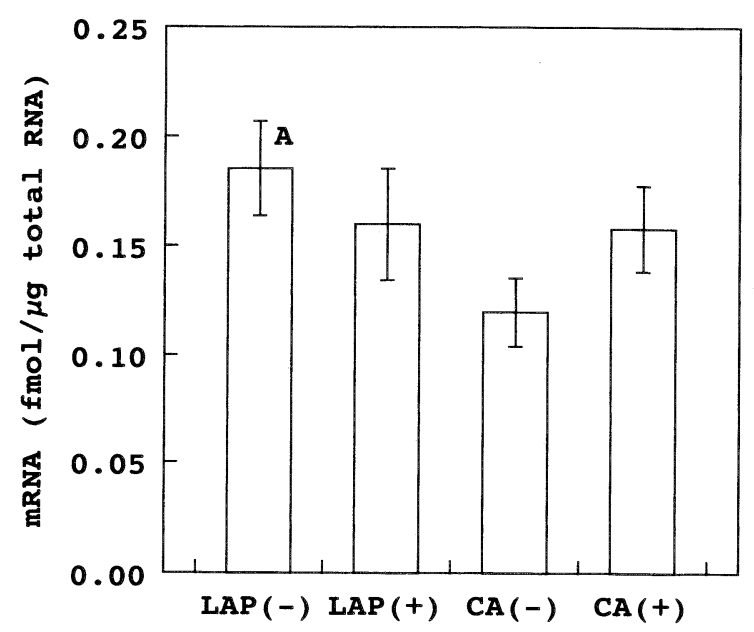

Fig. 2. Effects of cholesterol feeding on the expression of hepatic cholesterol $7 \alpha$-hydroxylase mRNA. Data are mean \pm SE of 7 quail. The uppercase letter (A) denotes a statistically significant difference between strains at $p<0.05$.

bile acid (Fig. 1 and Table 1), it is possible to estimate the total steroid (cholesterol + bile acid) retained by both LAP and CA quail during the period of cholesterol feeding. The total cholesterol retained in the body throughout $14 \mathrm{~d}$ of the experiment was $157.1 \mathrm{mg}$ for LAP quail and $62.8 \mathrm{mg}$ for CA quail. The LAP strain thus accumulated $94.3 \mathrm{mg}$ of cholesterol more than the CA quail, and this was equivalent to the accumulation of $6.7 \mathrm{mg} /$ d. A large part of the cholesterol accumulation was explained by the increased absorption of dietary cholesterol as described in the Results section $(4.5 \mathrm{mg} / \mathrm{d})$. The LAP strain excreted less bile acid into the feces than the CA quail did. The difference in the bile acid excretion thus proves that the rest of the cholesterol accumulated in the LAP strain. The higher capability of lipid absorption in the LAP quail therefore may reasonably explain their higher susceptibility to experimental atherosclerosis.

The total cholesterol administered was $700 \mathrm{mg} /$ quail during the $14 \mathrm{~d}$ of this experiment. The apparent absorption rate was $31.6 \%$ for LAP quail and $22.7 \%$ for CA quail, respectively. The difference in the apparent absorption rate thus appeared to be small and much less significant in this sense. The difference was however substantial from the quantitative view, taking into consideration the limited cholesterol pool size of Japanese quail as discussed below.

The mean total volume of serum for the LAP and CA quail was estimated to be 6.7 and $7.3 \mathrm{~mL}$, respectively, based on the assumption that the blood comprised $10 \%$ of the body weight and the hematocrit value $(32 \%)$ of chicken. Thus, the difference in the cholesterol absorption described above was very significant to the limited serum pool size of Japanese quail. Of the total cholesterol $(157.1 \mathrm{mg})$ retained by LAP quail, the serum $(90.5 \mathrm{mg})$ and liver $(24.9 \mathrm{mg})$ may account for 57.6 and $15.8 \%$, respectively. (Pool size for the liver was calculated on the basis of the assumption that the liver weight is $1.7 \mathrm{~g} / 100 \mathrm{~g}$ body weight. The liver weight of quail is usually in a range between 1.5 to $2.0 \mathrm{~g} / 100 \mathrm{~g}$ body weight). The rest of the cholesterol $(41.7 \mathrm{mg})$ not explained by these two major pools may be retained in the extra hepatic tissues such as adipose tissues and muscles, and/or leave the body in the form of feather debris. This may inversely suggest that Japanese quail have an extra pool capable of accommodating a maximum of about $40 \mathrm{mg}$ of cholesterol under atherogenic conditions. This postulate appeared to be true for the case of CA quail fed cholesterol. Of the cholesterol retained in the CA quail $(62.8 \mathrm{mg}), 39.5 \mathrm{mg}$ was localized in the extra pool, and $18.1 \mathrm{mg}$ and $5.2 \mathrm{mg}$ respectively in the serum and liver.

The feeding of cholesterol had no effect on the expression of hepatic cholesterol $7 \alpha$-hydroxylase mRNA in LAP quail. The LAP strain appeared to lack the upward regulation of this enzyme by dietary cholesterol (13). This lack of regulation may also contribute to some extent to the higher susceptibility of this strain to atherosclerosis. It has been suggested that the activity of cholesterol $7 \alpha$-hydroxylase is considerably lower in hyperresponsive pigeons than in hyporesponsive pigeons, which reasonably explains the hypercholesterolemia of this strain (14). Thus, the reduced rate of cholesterol catabolism of the LAP strain under atherogenic conditions may similarly aggravate hypercholesterolemia.

The LAP quail are unique among the experimental animals in that they easily develop atherosclerosis due to increased cholesterol absorption. Although more cytogenetic and physiological experiments are needed before a definitive factor for the increased cholesterol absorption is known, we have found the LAP quail to be of great value in the study of the relationship between atherogenesis and cholesterol absorption.

\section{REFERENCES}

1) Nagata J, Oku H, Toda T, Chinen I. 1996. Effect of dietary cholesterol on the activities of key enzymes of cholesterol metabolism in hyperlipidemia- and atherosclerosis-prone Japanese quail. J Nutr Sci Vitaminol 42: 287-300.

2) Iwasaki H, Oku H, Toda T, Nasu T, Oda K, Miyagi T, Chinen I. 1999. Apolipoprotein A-I of hyperlipidemia atherosclerosis prone (LAP) quail: cDNA sequence and tissue expression. Biosci Biotechnol Biochem 63: 29-34.

3) Iwasaki H, Oku H, Toda T, Nasu T, Miyagi T, Chinen I. 2000. The major low molecular weight apolipoprotein from normal and hyperlipidemia atherosclerosis-prone (LAP) Japanese quail. Biochim Biophys Acta 1483: 316-324.

4) Nagata J, Maeda G, Oku H, Toda T, Chinen I. 1997. Lipoprotein and apoprotein profiles of hyperlipidemic atherosclerosis-prone Japanese quail. J Nutr Sci Vitaminol 43: 47-57.

5) Allain CC, Poon LS, Chan CS, Richmond W, Fu PC. 1974. Enzymatic determination of total serum cholesterol. Clin Chem 20: 470-475.

6) Eaton DL, Klaassen CD. 1976. Effects of acute administration of taurocholic and taurochenodeoxycholic acid on biliary lipid excretion in the rat. Proc Soc Exp Biol Med 151: 198-202.

7) Van de Kamer JH, Huinink T, Weiyers HA. 1949. Rapid 
method for the determination of fat in feces. J Biol Chem 177: 347-355.

8) Salyers AA, Sperry JF, Wilkins TD, Walker AR, Richardson NJ. 1977. Neutral steroid concentrations in the faeces of North American White and South African Black populations at different risks for cancer of the colon. S Afr Med J 51: 823-827.

9) Fletcher MJ. 1972. Standardization of triglyceride methodology. Ann Clin Lab Sci 2: 389-392.

10) Rouser G, Simon G, Kritchevsky G. 1969. Species variations in phospholipid class distribution of organs. I. Kidney, liver and spleen. Lipids 4: 599-606.

11) Oku H, Toda $T$, Nagata J, Ishikawa $M$, Neyazaki $K$, Shinjyo C, Chinen I. 1997. Apolipoprotein A-1 of Japanese quail: cDNA sequence and modulation of tis- sue expression by cholesterol feeding. Biosci Biotechnol Biochem 61: 286-290.

12) Snedecor GW, Cochran WG. 1980. Two-way tables with unequal numbers and proportions. In: Statistical Methods (7th ed.), the Iowa State University Press, p 414-433. Ames, Iowa, USA.

13) Li YC, Wang DP, Chiang JY. 1990. Regulation of cholesterol 7 alpha-hydroxylase in the liver. Cloning, sequencing, and regulation of cholesterol 7 alpha-hydroxylase mRNA. J Biol Chem 265: 12012-12019.

14) Hulcher FH, Margolis R. 1982. Rate limiting, diurnal activity of hepatic microsomal cholesterol $7 \alpha$-hydroxylase in pigeons with high serum cholesterol. Biochim Biophys Acta 71 2: 242-249. 